\title{
Autodépistage du VIH au Canada : vision et plan d'action
}

\author{
Nitika Pant Pai MD MSP PhD, Réjean Thomas MD
}

Citation : CMAJ 2020 November 2;192:E1367-8. doi : 10.1503/cmaj.201160-f

Voir la version anglaise de l'article ici : www.cmaj.ca/lookup/doi/10.1503/cmaj.201160

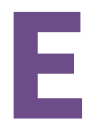

n 2020, comparativement aux cibles 95-95-95 pour l'élimination du VIH (95\% de dépistage, $95 \%$ de traitement et $95 \%$ de rétention en traitement) du Programme commun des Nations Unies sur le VIH/sida (ONUSIDA), le Canada avait un taux de $86 \%$ pour le dépistage, de $81 \%$ pour le traitement et de $91 \%$ pour la rétention en traitement $^{1}$. Malgré l'accessibilité du dépistage, $14 \%-20 \%$ des Canadiens vivant avec le VIH ignorent qu'ils sont infectés ${ }^{2}$. La non-détection du VIH est élevée dans certaines populations, comme les Autochtones, les consommateurs de drogues injectables, les immigrants et les hommes ayant des relations sexuelles avec des hommes. Selon les dernières données québécoises en matière de surveillance du VIH, 25,5\% des personnes qui ont reçu un diagnostic d'infection par le VIH en 2018 avaient un taux de CD4 inférieur à 200/ $\mathrm{mm}^{3}$.

Récemment, le gouvernement canadien a annoncé le lancement d'un plan d'action quinquennal sur la lutte contre le VIH et les infections transmissibles sexuellement et par le sang (ITSS), qui fait suite à la publication d'un cadre pancanadien encourageant l'adoption d'approches axées sur la personne pour améliorer l'accès et le recours au dépistage du VIH et des ITSS chez les populations clés. L'une des stratégies qui favoriseraient l'élimination du VIH au Canada est l'autodépistage de l'infection ${ }^{3}$.

L'autodépistage du VIH (ADVIH) est une stratégie dans laquelle une personne prélève elle-même son échantillon de salive, de sang ou d'urine, réalise un test de dépistage du VIH, interprète et consigne les résultats, et cherche proactivement à subir un test de confirmation et à obtenir du counseling et des soins ${ }^{4}$. Tout test d'autodépistage réactif ou positif nécessite une confirmation en laboratoire.

À l'échelle mondiale, 2 stratégies d'ADVIH ont été déployées : I'ADVIH assisté ou supervisé, où les participants sont encadrés dans un milieu de soin par des conseillers, des infirmières ou des pairs travailleurs de la santé, ainsi que l'ADVIH non assisté ou non supervisé, où les participants réalisent leur test de façon indépendante dans un endroit privé (domicile, bureau, comptoir

\section{POINTS CLÉS}

- L'autodépistage du VIH (ADVIH) est efficace et utilisé dans de nombreux pays.

- Le Canada tire de l'arrière en ne rendant pas l'ADVIH largement accessible.

- L'approbation rapide de tests de salive, de sang ou d'urine éprouvés et abordables favorisant l'autodépistage chez les populations clés est nécessaire pour permettre au Canada d'atteindre ses cibles en matière de dépistage du VIH.

- L'ADVIH devrait être intégré aux programmes de dépistage provinciaux et aux modèles de prestation de service qui sont axés sur les utilisateurs et qui font appel aux pharmacies, à des applications en ligne ou mobiles, à des machines distributrices ou à des organismes communautaires.

de service) et communiquent proactivement avec des conseillers et des cliniques pour obtenir des soins.

Dans ses lignes directrices, l'Organisation mondiale de la Santé présente l'ADVIH comme une solution de rechange au dépistage classique en laboratoire ${ }^{4}$. Plus de 250 études menées un peu partout dans le monde ont montré que l'ADVIH améliore l'accès et le recours au dépistage du VIH et accroît la fréquence de test, le dépistage des partenaires et l'orientation des amis et de la famille ${ }^{5}$. Sa rentabilité dans divers milieux partout sur la planète a également été prouvée ${ }^{6}$. En outre, l'ADVIH peut augmenter la demande de services connexes chez les personnes qui obtiennent un résultat négatif, services qui comprennent notamment l'accès à une prophylaxie préexposition (PPrE), l'orientation des partenaires et l'envoi d'alertes à ces derniers (résultats du test, PPrE et services).

L'ADVIH est maintenant offert, entre autres, au RoyaumeUni, en France, aux Pays-Bas, aux États-Unis, en Lettonie, en Espagne, au Kenya et en Afrique du Sud ${ }^{3}$. Des lignes directrices sur le sujet ont été rédigées dans plus de 60 pays $^{3}$. Par ailleurs, la disponibilité de l'ADVIH a accru la demande de modèles de prestation de service novateurs visant par exemple l'offre de 
counseling par l'intermédiaire d'applications pour téléphones intelligents ou de sites Web ${ }^{7,8}$, la distribution de médicaments en pharmacie ou par des machines distributrices, et la prestation de soins de suivi par des pairs conseillers en milieu communautaire.

Au Canada, il n'y a actuellement aucun test d'autodépistage approuvé, aucune stratégie d'ADVIH et aucune ligne directrice provinciale ou fédérale en la matière. Parmi les raisons pouvant expliquer ce retard figurent le manque de financement et de volonté politique, et la surveillance du dépistage du VIH par les laboratoires de santé publique.

En prévision de l'approbation imminente de tests d'autodépistage du VIH avec un échantillon de salive ou de sang par Santé Canada ${ }^{10}$, nous mettons en évidence 4 facteurs importants pour l'introduction optimale de l'ADVIH au Canada.

Premièrement, il existe un certain nombre de tests d'autodépistage du VIH préqualifiés par l'Organisation mondiale de la Santé, certifiés CE (ce qui indique la conformité des produits vendus dans l'Espace économique européen aux normes en matière de santé, de sécurité et de protection de l'environnement), ou approuvés par le Secrétariat américain aux produits alimentaires et pharmaceutiques (FDA) des États-Unis. L'approbation de ces tests au Canada devrait être envisagée ${ }^{11}$. Qui plus est, l'approbation rapide de tests d'autodépistage reposant sur l'analyse d'un échantillon de salive, de sang ou d'urine pourrait donner plus d'options de dépistage, accroître la concurrence et réduire le coût unitaire des tests, favorisant ainsi leur abordabilité et leur utilisation par les populations prioritaires. Comme les résultats positifs à l'ADVIH nécessitent une confirmation rapide en laboratoire, ils devront être intégrés aux algorithmes de dépistage du VIH existants.

Deuxièmement, la commercialisation à grande échelle de tests d'autodépistage abordables devrait stimuler la création de modèles de prestation de service axés sur les utilisateurs. Ces modèles pourraient être autonomes ou intégrés aux programmes de dépistage existants, et pourraient être déployés dans des centres de proximité, des unités mobiles, des comptoirs de service, des cliniques privées, des machines distributrices, des applications pour téléphones intelligents et des organismes de sensibilisation. L'adaptation des modèles de dépistage du VIH actuels pour diriger les utilisateurs vers des soins cliniques devrait améliorer la qualité, l'efficacité et la pérennité, mais nécessitera l'établissement d'une collaboration continue avec les fournisseurs privés, les pharmacies, les organismes communautaires, les cliniques publiques, les fabricants de tests et les laboratoires ${ }^{12}$.

Troisièmement, la prise en compte de l'aspect économique de l'intégration de l'ADVIH est essentielle à la pérennité. Les systèmes de santé du Canada, en grande partie gérés par les provinces, combinent soins publics et soins privés. L'intégration réussie des services d'ADVIH exigera l'utilisation de divers modèles de financement publics et privés; par exemple, on pourrait mettre en place un système de remboursement public pour les populations à faible revenu et un système de paiement privé pour celles à revenu élevé. Les trousses d'autodépistage pourraient coûter entre 5 \$CA et 15 \$CA, comme les tests de grossesse. Parmi les autres services nécessaires à la mise en œuvre optimale de l'ADVIH au Canada, notons l'orientation des personnes ayant obtenu un résultat positif à l'autodépistage vers un laboratoire pour qu'elles subissent un test de confirmation, l'offre de counseling, le traitement par un fournisseur, et les services de prévention comme la PPrE, l'orientation des partenaires et l'envoi d'avis aux personnes ayant obtenu un résultat négatif.

Et quatrièmement, des essais à grande échelle et des études de rentabilité sur la mise en œuvre de l'ADVIH au Canada sont requis pour guider l'élaboration de politiques et de lignes directrices fédérales et provinciales en la matière.

L'introduction de I'ADVIH au Canada devrait améliorer la détection du VIH chez les populations où le dépistage est insuffisant en raison de la présence d'obstacles, ainsi que la prise en charge de l'infection. Cela nous rapprocherait des cibles 95-95-95 de l'ONUSIDA et nous permettrait de franchir une étape historique dans la longue lutte contre l'épidémie de VIH au pays.

\section{Références}

1. Canada's progress on 90-90-90 targets [affiche infographique]. Toronto: Canadian AIDS Treatment Information Exchange; 2018. Accessible ici : library. catie.ca/pdf/ATI-40000s/40264.pdf (consulté le 25 octobre 2019).

2. The epidemiology of HIV in Canada [fiche descriptive]. Toronto: Canadian AIDS Treatment Information Exchange; 2018. Accessible ici : www.catie.ca/sites/ default/files/fs-epi-hiv-canada-EN-2018-09-06.pdf (consulté le 25 octobre 2019).

3. HIVST.org (HIV self-testing research and policy hub) [page d'accueil]. Accessible ici : www.hivst.org/ (consulté le 25 octobre 2019).

4. Guidelines on HIV self-testing and partner notification: supplement to consolidated guidelines on HIV testing services. Geneva: World Health Organization; 2016. Accessible ici : apps.who.int/iris/bitstream/handle/10665/251655 /9789241549868-eng.pdf?sequence=1 (consulté le 25 octobre 2019).

5. Indravudh PP, Choko AT, Corbett EL. Scaling up HIV self-testing in sub-Saharan Africa: A review of technology, policy and evidence. Curr Opin Infect Dis 2018;31:14-24.

6. Cambiano V, Johnson CC, Hatzold K, et al. Working Group on Cost Effectiveness of HIV self-testing in Southern Africa. The impact and cost-effectiveness of community-based HIV self-testing in sub-Saharan Africa: a health economic and modelling analysis. J Int AIDS Soc 2019;22(Suppl 1):e25243.

7. Pant Pai N, Smallwood M, Desjardins L, et al. An unsupervised smart appoptimized HIV self-testing program in Montreal, Canada: cross-sectional study. $J$ Med Internet Res 2018;20:e10258.

8. Pant Pai N, Bhargava M, Joseph L, et al. Will an unsupervised self-testing strategy be feasible to operationalize in Canada? Results from a pilot study in students of a large Canadian university. Aids Res Treat 2014;2014:747619.

9. Innovative WHO HIV testing recommendations aim to expand treatment coverage [communiqué de presse]. Geneva: World Health Organization; 2019 Nov. 19. Accessible ici : www.who.int/news-room/detail/27-11-2019-innovative -who-hiv-testing-recommendations-aim-to-expand-treatment-coverage (consulté le 22 septembre 2020).

10. Mitchell A. Why it's time for Canada to introduce self-testing HIV kits. Macleans 2020 Jan. 16. Accessible ici : www.macleans.ca/education/college/ why-its-time-for-canada-to-introduce-self-testing-hiv-kits/ (consulté le 23 septembre 2020).

11. Reaching the undiagnosed: HIV self-testing in Canada - What should we expect? Webinar series: Reaching the undiagnosed. Toronto: Canadian AIDS Treatment Information Exchange; 2018 Mar. 28. Accessible ici : www.catie.ca/en/webinars/ reaching-undiagnosed-hiv-self-testing-canada-what-should-we-expect (consulté le 22 septembre 2020).

12. Pai NP, Smallwood M, Gulati D, et al. What do key stakeholders think about HIV self-testing in Canada? Results from a cross-sectional survey. AIDS Behav 2018;22:606-15. 
Intérêts concurrents : Nitika Pant Pai a reçu une subvention du gouvernement du Canada (Grands Défis Canada) pour HIVSmart! (droit d'auteur $n^{\circ}$ 1105598, Université McGill, 2013), une application et un programme numériques mondiaux libres d'accès pour l'autodépistage du VIH. Réjean Thomas a reçu des honoraires de Gilead Sciences, de Merck et de ViiV Healthcare, pour des présentations à des congrès et des participations à des conseils consultatifs.

Cet article a été révisé par des pairs.

Affiliations : Département de médecine (Pant Pai), Université McGill; Institut de recherche du Centre universitaire de santé McGill (Pant Pai); clinique médicale l'Actuel (Thomas), Montréal (Qc).

Collaborateurs : Les deux auteurs ont contribué à la conception du travail, ont rédigé le manuscrit et en ont révisé de façon critique le contenu intellectuel important; ils ont donné leur approbation finale pour la version destinée à être publiée et assument l'entière responsabilité de tous les aspects du travail.
Financement : Nitika Pant Pai souligne le soutien sous forme de subventions de fonctionnement reçu des Instituts de recherche en santé du Canada (HHP-137872 et PJT-153149) et du Fonds de recherche Santé Québec (chercheurs-boursiers Senior).

Propriété intellectuelle du contenu : Il s'agit d'un article en libre accès distribué conformément aux modalités de la licence Creative Commons Attribution (CC BY-NC-ND 4.0), qui permet l'utilisation, la diffusion et la reproduction dans tout médium à la condition que la publication originale soit adéquatement citée, que l'utilisation se fasse à des fins non commerciales (c.-à-d., recherche ou éducation) et qu'aucune modification ni adaptation n'y soit apportée. Voir : https://creativecommons. org/licenses/by-nc-nd/4.0/.

Remerciements : Les auteurs remercient Nandi Belinsky et Angela Karellis pour leur aide avec la mise en forme et les références.

Correspondance : Nikita Pant Pai, nitika.pai@mcgill.ca 